\title{
Scattering mechanisms of electrons in monocrystalline PbTe, PbSe and PbS
}

\author{
D.M. Freik, L.I. Nykyruy, V.M. Shperun \\ Physics-chemical institute at the Vasyl Stefanyk Precarpathian University, \\ 57 Shevchenko Str., 76025 Ivano-Frankivsk, Ukraine, \\ E-mail:freik@pu.if.ua
}

\begin{abstract}
The theoretical analysis of carrier scattering mechanisms in electronic lead chalcogenide crystals was carried out. The calculation of carrier mobility in wide temperature $(4.2-300 \mathrm{~K})$ and concentration $\left(10^{16}-10^{20} \mathrm{~cm}^{-3}\right)$ ranges is carried out from the viewpoint of interaction of conductivity electrons with deformation potentials of acoustic and optical phonons, polarizing potential of optical phonons, screening Coulombic and short-range potentials of vacancies. It has been shown that the agreement of theoretical and experimental results takes place when taking into account the carrier scattering both on phonons and ionized vacancies.
\end{abstract}

Keywords: vacancies, optical and acoustic phonons, lead chalcogenides, scattering mechanism, interaction.

Paper received 03.09.02; accepted for publication 17.12.02.

\section{Introduction}

The exceeding scattering mechanisms in lead chalcogenides crystals of $\mathrm{n}$-type conductivity were investigated in various papers [1-25], but the separation and difference at the obtained results requires an essential correction. The analysis of experimental data upon transport phenomena (mobility, thermal-e.m.f., Nernst-Ettinhshausen effect, to an electronic thermal conductivity) shows that, in lead chalcogenide crystals the carrier scattering caused by interaction with proper point defects and thermal oscillations of a crystalline lattice [1-7] are dominating. The relative contribution of these mechanisms is reduced to the following.

According to majorities of the researches carried out, in the very wide temperature range (beginning from $1 \mathrm{~K}$ and to higher), with the greater or smaller exactness, it is possible to limit ourselves by the carrier scattering on acoustic phonons [1-10]. This conclusion is made by comparison between calculated and experimental dependences of kinetic coefficients on temperature and concentration. In accord to [1,11], the mobility limited by scattering on acoustic phonons should vary under the $T^{-5 / 2}$ law, which is observed in experiment. The same conclusion about significant role of scattering electrons on acoustic phonons, down to low temperature fields, indicate existence of superconductivity properties of $\mathrm{A}^{\mathrm{IV}} \mathrm{B}^{\mathrm{VI}}$ compounds [8,9]. In the vicinity of the room temperature, the analysis of data on thermoelectromotive is possible to explain it by a combination of scattering on acoustic and optical phonons $[1,5,8,9]$.

The scattering on optical phonons is considered taking into account two components: polar and deformational optical phonons.

The scattering on short-wave phonons with passage of carriers between equivalent valleys is an improbable process for electronic crystals. This aspect of scattering is realized with a higher probability in lead chalcogenides of the $p$-type conductivity [1].

In the field of low temperatures, the carriers of a current are dispersed mainly on charged vacancies [1,2]. Both the scattering on screening Coulomb potential of vacancies predominates in the field of low concentration and dominating becomes scattering carriers on a shortrange potential of vacancies (interior part of Coulomb potential, which operates on lengths close to a constant of lattice) in the field of high concentration.

The exceeding of scattering mechanisms in semiconductors, as a rule, determine from associations of relaxation time from an energy, carriers concentration, or tem- 


\section{D.M. Freik et al.: Scattering mechanisms of electrons in ...}

perature $[1,2]$. At a simultaneous realization of different alternative mechanisms scattering the summarized relaxation time is determined by Mattisen rule [13]:

$$
\tau^{-1}=\sum_{i} \tau_{i}^{-1}
$$

where $\tau_{i}$ is relaxation time for $\mathrm{i}$-type mechanism of scattering.

At the calculated used Cane's laws of dispersion:

$$
\begin{aligned}
& \varepsilon(k)=\frac{\hbar^{2} k^{2}}{2 m^{*}}, \\
& \varepsilon(k)=\frac{\hbar^{2} k^{2}}{2 m^{*}}+\frac{1}{2} \varepsilon_{G}+\sqrt{\varepsilon_{G}^{2}+\frac{4 \hbar^{2}}{m^{* 2}} k^{2} p^{2}},
\end{aligned}
$$

where (2) correspond to parabolic law of dispersion and (3) correspond of nonparabolic law of dispersion, accordingly. At these expansion $k$ is the wave vector, $\hbar$ is Plank's constant, $m^{*}$ - the effective mass of the charge carrier, $\varepsilon_{G}$ is forbidden gap.

The analyses of received results led from compare theoretical and experimental data of current mobility, what calculated by:

$$
\mu_{H}=\frac{e}{m^{*}} \tau I_{n, k}^{m}(z ; \beta)
$$

where $I_{n, k}^{m}(z ; \beta)$ are two-parameter Fermi's integrals, tabulated in [13], $\tau$ is the relaxation time for each separate mechanism of scattering or when taking into account contribution of several alternative mechanisms simultaneously, according to (1), $e$ is the electron charge.

\section{Theoretical representations of carriers scattering}

\subsection{Relaxation time for scattering on acoustic phonons}

The relaxation time for electrons dispersed on the deformational potential of acoustic phonons, when using the Cane law of dispersion, is determined by the relation [4]:

$$
\tau_{a}(x)=\frac{\tau_{0, v}\left(x+\beta x^{2}\right)^{-1 / 2}}{(1+2 \beta x)\left[(1-A)^{2}-B\right]},
$$

where $\tau_{0, a}=2 \pi \hbar^{4} C_{l} / E_{o c}^{2}\left(2 m^{*} k_{0} T\right)^{3 / 2}$, $A=\beta x(1-K) /(1+2 \beta x), B=8 \beta x(1+\beta x) K / 3(1+2 \beta x)^{2}$.

Here $E_{a c}$ - deformational potential of the conductivity band, $C_{1}$ - combination of elastic constants, $K=1.5$ is ratio of deformational potentials of acoustic phonons for conductivity and valence bands, $m^{*}$ - carrier effec- tive mass, $T$-temperature, $k_{0}$ - the Boltzmann constant, $x=\varepsilon_{0} / k_{0} T$ - reduced energy, $\beta=\varepsilon / \varepsilon_{G}$ - parameter of nonparabolicity, $\varepsilon_{G}$ - width of forbidden band, $\varepsilon$ - carrier energy (according to the law of variance).

\subsection{The scattering of carriers on polar optical phonons}

In the case of a simple isotropic parabolic model, the relaxation time for polar longitudinal optical phonons has the form [5]

$\tau_{o p t}^{-1} \sim \frac{1}{v k^{2}} \int_{0}^{2 k} q d q$

where $v$ - velocity of carriers, if the integral takes into account all phonons with a wave vector $q$,

$\tau_{o p t}^{-1}=\frac{2 k_{0} T e^{2}\left(\varepsilon_{\infty}^{-1}-\varepsilon_{0}^{-1}\right)}{\hbar^{2} v}$,

where $\varepsilon_{\infty}$ and $\varepsilon_{0}$ - high-frequency and static dielectric constants, accordingly. The difference $\varepsilon_{\infty}^{-1}-\varepsilon_{0}^{-1}$ is determined by polarity of crystal and determines a longwave part of a potential polar mode of lattice oscillations.

Significant difference between values $\varepsilon_{0}$ and $\varepsilon_{\infty}$ is motive necessity of the registration of screening effects [1-4].

The registration screening effects is carried out with the factor [5]

$1-\delta_{\infty} \ln \left(1+\delta_{\infty}^{-1}\right)$

where $\delta_{\infty}=\left(2 k r_{\infty}\right)^{-2}$

The account of nonparabolicity in the two-band model gives the following expression for the radius of screening $r_{\infty}$

$r_{\infty}^{-2}=\frac{2^{5 / 2} e^{2} m_{d}^{* 3 / 2}}{\pi \hbar^{3} \varepsilon_{\infty}} \int_{0}^{\infty}\left(-\frac{\partial f}{\partial \varepsilon}\right)\left(\varepsilon+\frac{\varepsilon^{2}}{\varepsilon_{G}}\right)^{1 / 2}\left(1+2 \frac{\varepsilon}{\varepsilon_{G}}\right) d \varepsilon$

In cases that concern values $k r_{\infty} \approx 1$, the screening effects carry on to a diminution of appropriate values of a relaxation time by the indicated factor twice. Besides the power association of a relaxation time also varies through a power association $\delta_{\infty}$.

Combining the screening effects and nonparabolic ones, the authors $[1,5]$ have received an expression of a relaxation time for the case of polar scattering

$$
\begin{aligned}
& \tau_{o p t}^{-1}=\frac{2^{1 / 2} e^{2} k_{0} T m_{d 1}^{* 1 / 2}}{\hbar^{2} \varepsilon^{1 / 2}}\left(\varepsilon_{\infty}^{-1}-\varepsilon_{0}^{-1}\right) \frac{1+\left(2 \varepsilon / \varepsilon_{G}\right)}{\left[1+\left(\varepsilon / \varepsilon_{G}\right)\right]^{1 / 2}} \times \\
& \times\left\{\left[1-\delta_{\infty} \ln \left(1+\delta_{\infty}^{-1}\right)\right]-\right. \\
& \left.-\frac{2 \varepsilon\left(\varepsilon_{G}+\varepsilon\right)}{\left(\varepsilon_{G}+\varepsilon\right)^{2}}\left[1-2 \delta_{\infty}+2 \delta_{\infty}^{2} \ln \left(1+\delta_{\infty}^{2}\right)\right]\right\} .
\end{aligned}
$$


In the formulae that determine polar optical phonons, there are no adjustable parameters, therefore, their contribution can be estimated rather precisely.

\subsection{Scattering on polar optical phonons at the Debye temperature}

Near Debye temperatures, relaxations cannot be used. However, account of nonparabolicity and the use of a quasielastic approximation gives the following expression for the mobility [3]

$$
\mu=\frac{k_{F} \hbar^{3}\left(e^{z}-1\right)^{2}}{2 e k_{0} \operatorname{Tm}_{0}^{* 2}\left(1+\frac{2 \varepsilon_{F}}{\varepsilon_{G}}\right)\left(\varepsilon_{\infty}^{-1}-\varepsilon_{0}^{-1}\right) e^{z} z^{2} d_{00}}
$$

Such labels are used here:

$$
\begin{aligned}
& d_{00}=S_{1}-\frac{2 \varepsilon_{F}\left(\varepsilon_{G}+\varepsilon_{F}\right)}{\left(\varepsilon_{G}+2 \varepsilon_{F}\right)^{2}} S_{2}, \\
& S_{1}=\frac{\left(e^{z}-1\right)^{2}}{e^{z}\left(2 k_{F} r_{e}\right)^{2}} \int_{0}^{\left(2 k_{F} r_{e}\right)^{2}} \frac{\exp \left[z\left(\frac{t}{t+1}\right)^{1 / 2}\right]\left(\frac{t}{t+1}\right)^{2} d t}{\left\{\exp \left[z\left(\frac{t}{t+1}\right)^{1 / 2}\right]-1\right\}^{2}} .
\end{aligned}
$$

$S_{2}$ is obtained with $S_{1}$ by multiplication integral of expression on $\frac{2 t}{\left(2 k_{F} r_{e}\right)^{2}}, \varepsilon_{F}$ - carriers energy on the Fermi level. The changeable integration $t$ arises through replacement of an element of integration for $q$ on $t=\left(q r_{e}\right)^{2}$. Parameter $k_{F}=\left(\frac{3 \pi^{2} n}{N}\right)_{1 / 2}^{1 / 3}-$ wave vector on the Fermi level, $r_{e}=\left(\frac{\varepsilon_{\infty}}{4 \pi e^{2} \rho\left(\varepsilon_{F}\right)}\right)^{1 / 2}$-radius of screening, $\rho\left(\varepsilon_{F}\right)$-density of states on the Fermi level (in all ellipsoids).

\subsection{Scattering of carriers on the deformational potential of optical phonons}

To recent time deformational optical phonons in crystals lead chalcogenides were not practically taken into account. For the first time, some attention was paid to them by Zayachuk [2] when analyzing the carrier mobility in $n$-PbTe crystals at $300 \mathrm{~K}$. He found that this mechanism can be dominating in a definite concentration range.

A possible contribution of the deformational potential of optical phonons can be represented according to (3), using relations for $\tau_{0}$ [2] in the following form: $\tau_{0, o}=2 \hbar^{2} \alpha^{2} \rho\left(\hbar \omega_{0}\right)^{2} / \pi\left(2 m_{n} k_{0} T\right)^{3 / 2} E_{o c}^{2}$,

where $K_{0}=E_{o v} / E_{o c}, \alpha$ is the lattice constant, $\rho-$ density of crystal, $\omega_{0}$ - frequency of an optical phonon, $E_{o v}$ and $E_{o c}$ - constants of deformational potential for valence and conductivity bands, accordingly.

\subsection{Scattering of carriers on screening Coulomb potential of vacancies}

The relaxation time of carrier scattering on the screening Coulomb potential inherent to vacancies can be calculated according to [2]:

$\tau_{\text {Coul }}(x)=\frac{\varepsilon_{0}^{2}\left(2 m^{*}\right)^{1 / 2}\left(k_{0} T\right)^{3 / 2}}{\pi\left(Z e^{2}\right)^{2} N_{v}[\operatorname{Ln}(1+\xi)-\xi /(1+\xi)]} \times$

$\times \frac{\left(x+\beta x^{2}\right)^{3 / 2}}{(1+2 \beta x)}$,

where $\xi=\left(2 k r_{v}\right)^{2}, k$ is the wave vector of carriers, $r_{v}-$ is radius of screening of vacancies potential, $x=\varepsilon / k_{0} T-$ reduced energy, $\beta=\varepsilon / \varepsilon_{G}$ - parameter of nonparabolic. The concentration of vacancies is assumed to be equal $N_{v}=2 \cdot 10^{18} \mathrm{~cm}^{-3}$ for $n<4 \cdot 10^{18} \mathrm{~cm}^{-3}$, and $N_{v}=n / 2$ for $n \geq 4 \cdot 10^{18} \mathrm{~cm}^{-3}$.

\subsection{Scattering of carriers on the short-range potential of vacancies}

The scattering of carriers on a short-range potential of vacancies, as well as scattering on deformational potential of acoustic phonons, can be expressed as in (4) with a replacement $\tau_{0 a}$ on $\tau_{\text {core }}$ :

$\tau_{0, \text { core }}=\pi \cdot \hbar^{4} / m^{*}\left(2 m^{*} k_{0} T\right)^{1 / 2} U_{V C}^{2} N_{v}$.

\section{Results}

From Figs 1, 2 and Table one can see that the account of all mentioned scattering mechanisms gives a good accordance between theoretical and experimental results, and correctly describes appearances of transposition in lead chalcogenides electronic crystals.

Carrier scattering on vacancies (Fig. 1, curves 2, 3, Fig. 2, curves 3, 4, Fig. 2, curve 5, table) and their shortrange potential in the part that operates on lengths close to the lattice constant (Fig. 1, Fig. 2, curve 1, the table)) gives a noticeable contribution at the temperatures $77-$ $300 \mathrm{~K}$ only in the of high concentrations $\left(\sim 10^{19} \mathrm{~cm}^{-3}\right.$ and higher). At the liquid helium temperature, scattering on vacancies is dominating and to a great extent determines appearances of transposition. Such conclusion coincides with results of antecedent researches, in particular, with those by Ravych [3,4] and Zayachuk [2]. The scattering 


\section{D.M. Freik et al.: Scattering mechanisms of electrons in ...}
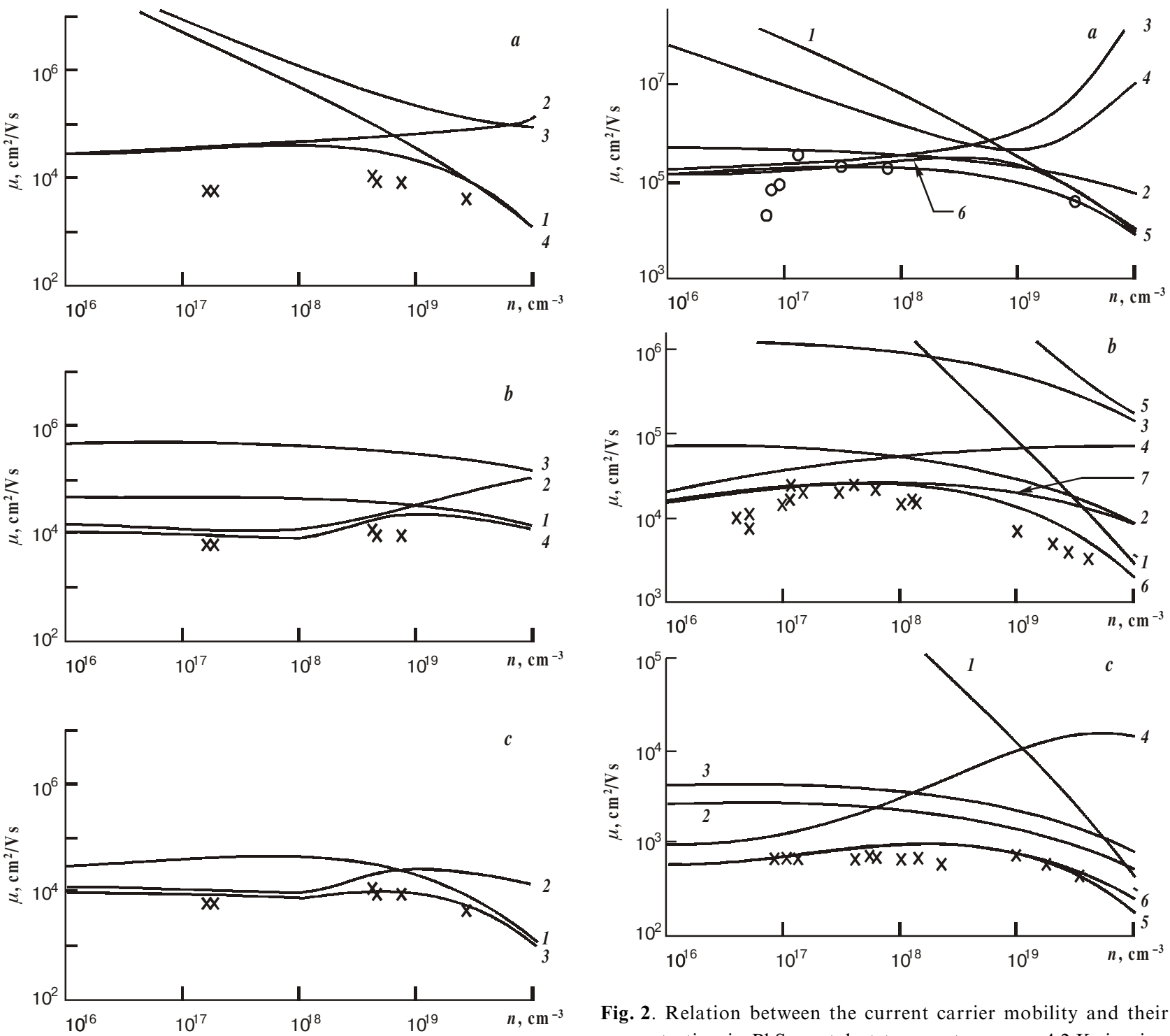

Fig. 2. Relation between the current carrier mobility and their concentration in PbS crystal at temperatures: $a-4.2 \mathrm{~K}$, in view of scattering free carriers on: $l$ - short-range potential of vacancies, 2 - deformational potential of acoustic phonons (and together with it summarized scattering on oscillations crystalline lattice), 3 - coulomb potential of vacancies (at $N_{v}=2 \cdot 10^{18} \mathrm{~cm}^{-3}$ ), 4 - Coulomb potential of vacancies (at $N_{v}=n / 2$ ), 5 - simultaneous registration of all aspects of scattering, 5 - summarized scattering on vacancies (till a curve $1,3,4) ; b-77 \mathrm{~K}$, in view of scattering carriers on: $l$ - short-range potential of vacancies (and together with its summarized scattering on vacancies), 2 deformational potential of acoustic phonons, 3 - deformational potential of optical phonons, 4 - polarizing potential of optical phonons; 5 - coulomb potential of vacancies (at $\left.N_{v}=2 \cdot 10^{18} \mathrm{~cm}^{-3}\right), 6-$ simultaneous registration of all aspects of scattering (till a curve $1-5$ ), 7 - summarized scattering of carriers on phonons (till a curve 2-4); $c-300 \mathrm{~K}$, in view of scattering carriers on: $l$ - short-range potential of vacancies (and together with it summarized scattering on vacancies), 2 - deformational potential of acoustic phonons, 3 - deformational potential of optical phonons, 4 - polarizing potential of optical phonons; 5 simultaneous registration of all aspects of scattering (till a curve 1-4), 6 - summarized scattering of carriers on phonons (till a curve 2-4). Experimental values are taken from $[15,16]$. 
D.M. Freik et al.: Scattering mechanisms of electrons in ...

Table. Concentration and temperature limits of dominant separate scattering mechanisms on lead chalcogenides electronic crystals

\begin{tabular}{|c|c|c|c|}
\hline & $4.2 \mathrm{~K}$ & $77 \mathrm{~K}$ & $300 \mathrm{~K}$ \\
\hline & \multicolumn{3}{|c|}{ Screening Coulomb potential of vacancies } \\
\hline $\mathrm{PbS}$ & $1 \cdot 10^{16}-5 \cdot 10^{18}, 5 \cdot 10^{18}-1 \cdot 10^{19}$ & $1 \cdot 10^{16}-5 \cdot 10^{17}$ & - \\
\hline $\mathrm{PbSe}$ & $1 \cdot 10^{16}-2 \cdot 10^{18}, 7 \cdot 10^{18}-1 \cdot 10^{19}$ & - & - \\
\hline \multirow[t]{2}{*}{$\mathrm{PbTe}$} & $1 \cdot 10^{17}-2 \cdot 10^{17}$ & - & - \\
\hline & \multicolumn{3}{|c|}{ Short-range potential of vacancies } \\
\hline $\mathrm{PbS}$ & $1 \cdot 10^{19}-1 \cdot 10^{20}$ & $2 \cdot 10^{19}-1 \cdot 10^{20}$ & $8 \cdot 10^{19}-1 \cdot 10^{20}$ \\
\hline $\mathrm{PbSe}$ & $2 \cdot 10^{19}-1 \cdot 10^{20}$ & $5 \cdot 10^{19}-1 \cdot 10^{20}$ & $8 \cdot 10^{19}-1 \cdot 10^{20}$ \\
\hline \multirow[t]{2}{*}{$\mathrm{PbTe}$} & $1 \cdot 10^{19}-1 \cdot 10^{20}$ & $8.5 \cdot 10^{19}-1 \cdot 10^{20}$ & $>1 \cdot 10^{20}$ \\
\hline & \multicolumn{3}{|c|}{ Polar potential of the optical phonons } \\
\hline $\mathrm{PbS}$ & - & $1 \cdot 10^{16}-8 \cdot 10^{18}$ & $1 \cdot 10^{16}-1 \cdot 10^{18}$ \\
\hline $\mathrm{PbSe}$ & - & $1 \cdot 10^{16}-8 \cdot 10^{17}$ & $1 \cdot 10^{16}-6 \cdot 10^{17}$ \\
\hline \multirow[t]{2}{*}{$\mathrm{PbTe}$} & - & $1 \cdot 10^{16}-8.5 \cdot 10^{17}$ & $1 \cdot 10^{16}-7 \cdot 10^{18}$ \\
\hline & \multicolumn{3}{|c|}{ Deformational potential of optical phonons } \\
\hline$\overline{\mathrm{PbS}}$ & - & - & $5 \cdot 10^{18}-1 \cdot 10^{20}$ \\
\hline $\mathrm{PbSe}$ & - & - & $1 \cdot 10^{19}-2,5 \cdot 10^{19}$ \\
\hline \multirow[t]{2}{*}{$\mathrm{PbTe}$} & - & - & $8 \cdot 10^{18}-1 \cdot 10^{20}$ \\
\hline & \multicolumn{3}{|c|}{ Deformational potential of acoustic phonons } \\
\hline $\mathrm{PbS}$ & $5 \cdot 10^{18}-5 \cdot 10^{19}$ & $1 \cdot 10^{16}-1 \cdot 10^{20}$ & $5 \cdot 10^{18}-1 \cdot 10^{20}$ \\
\hline$\overline{\mathrm{PbSe}}$ & $1 \cdot 10^{16}-8 \cdot 10^{19}$ & $1 \cdot 10^{17}-7 \cdot 10^{19}$ & $6 \cdot 10^{17}-1 \cdot 10^{20}$ \\
\hline $\mathrm{PbTe}$ & $1 \cdot 10^{16}-1 \cdot 10^{20}$ & $8 \cdot 10^{17}-1 \cdot 10^{20}$ & $\sim 1 \cdot 10^{20}$ \\
\hline
\end{tabular}

of carriers on polar optical phonons is dominating in the field of low and average concentrations at temperatures 77 and $300 \mathrm{~K}$ (Figs 1 and 2, curve 4, table). At higher carrier concentrations their influence on appearance of transposition decreases, and mechanisms of scattering on the deformational potential of acoustic and optical phonons predominate. The relative share of scattering on optical polar phonons decreases with lowering the temperature below the nitrogen one. At $T<10 \mathrm{~K}$ polar optical phonons are not excited, therefore polar scattering is insignificant. The account of this mechanism gives the actual agreement between the theory and experiment in the temperature range $20-300 \mathrm{~K}$. The essential role of optical polar phonons at these temperatures is confirmed by an experimentally observable diminution of the carrier mobility at low concentrations (Figs 1,2).

The carrier scattering on the deformational potential of optical phonons up to date was not practically taken into account, though, as was shown in [2], at $300 \mathrm{~K}$ it is rather essential. The researches carried out by us confirm the important role of this scattering mechanism at room temperatures for lead chalcogenide crystals. The use of expression (10) for a considered relaxation time in a combination with polar optical and acoustic phonons enables to reach the good agreement between the theory and experiment (Fig. 2, curve 3). With this purpose, to describe $\tau_{0}$, using the expressions (10) magnitude $E_{o c}$ should be taken equal to $26 \mathrm{eV}$. The contribution of the optical deformational potential, as seen from Fig. 2 (curve 3 ), at the room temperature is rather essential, and at high concentrations is even dominating in carrier mobility. At temperatures of liquid helium, scattering on optical deformational phonons is less essential (Fig. 1b, Fig. 2b,c, curve 3).

Acoustic phonons influence on kinetic parameters of lead chalcogenides crystals in all the considered temperature $(4.2-300 \mathrm{~K})$ and concentration $\left(10^{16}-10^{20} \mathrm{~cm}^{-3}\right)$ ranges. Let us note that, in the low concentration range, some deviation of experimental results from data of calculations (Fig. 1, 2) takes place. Therefore, it is possible to assume the necessity of considering inelastic effects when evaluating scattering mechanisms.

\section{Conclusions}

1. The dominating mechanisms of carrier scattering in electronic lead chalcogenide crystals are both scattering on vacancies and on thermal oscillations of the lattice. In the range of low temperatures, carrier scattering on vacancies predominates, while at high temperatures on thermal oscillations of crystalline lattice.

2. Role of optical polar phonons are significant at temperatures 77 and $300 \mathrm{~K}$ for concentrations $10^{16}$ 


\section{D.M. Freik et al.: Scattering mechanisms of electrons in ...}

$10^{18} \mathrm{~cm}^{-3}$. At magnification of concentration the scattering on optical phonons is hindered due to screening.

3. At high concentrations (higher than $10^{19} \mathrm{~cm}^{-3}$ ), the scattering on optical phonons is exhibited due to their deformational potential, the role of which in the total scattering at definite concentrations (see Table) becomes most essential at the room temperature, in particular, for lead sulphides and tellurides.

4. The scattering of carriers on acoustic phonons becomes essential at all considered temperatures in the whole researched concentration range. In the field of low concentrations at helium temperatures, for the agreement of theoretical calculations with experimental data, it is necessary to take into account inelastic effects at scattering of carriers on acoustic phonons.

5. The scattering on a short-range potential of vacancies is realized at high concentrations, and with increase of temperature the prevalence of this mechanism is displaced into the side of higher concentration values and decreases in the series PbS-PbSe-PbTe.

6. The Coulomb potential of vacancies dominates at low concentration and it payment in the total scattering, as in the case of a short-range potential of vacancies, decreases in the series $\mathrm{PbS}-\mathrm{PbSe}-\mathrm{PbTe}$.

\section{Reference}

1. Yu.I. Ravych, B.A. Efimova, I.A. Smirnov. Methods of research of semiconductor in application to lead chalcogenides PbSe, PbSe, PbS. Nauka, M. 384 p. (1968).

2. D.M. Zayachuk. To answer about predominant scattering mechanisms in lead telluride // Phys. Tech. Semicond. (Russia), 31(2), pp. 217-220 (1997).

3. Yu.I. Ravich, B.A. Efimova, V.I. Tamarchnko. Scattering of current carriers and transport phenomena in lead chalcogenides. I. Theory // Phys. Stat. Sol. (b), 43(1), pp. 1133 (1971).

4. Yu.I. Ravich, B.A. Efimova, V.I. Tamarchenko. Scattering of current carriers and transport phenomena in lead chalcogenides. II. Experiment // Phys. Stat. Sol. (b), 43(2), pp. 453-469 (1971).

5. Yu.I. Ravich, L.Ya. Morgovski. To scattering theory on optic and acoustic phonons in semiconductors of PbTe-like // Phys. Tech. Semicond. (Russia), 3(10),. cc. 1528-1539 (1969).

6. W. Szymanska. Zjawiska transportu elektronowego w chalcogenidkach oiowiu // Postery Fiziki, 26(1), pp. 5-16 (1975).

7. W. Zawadsky. Electron transport phenomena in small-gap semiconductors // Advances in physics, 23(3), pp. 435-522 (1974).

8. V.L. Volkov. Electron-phonon interaction on narrow-gap semiconductors // Phys. Tech. Semicond. (Russia), 12(2), pp. 396-398 (1978).

9. V.L. Volkov, I.V. Kucherenko, V.N. Moiseenko, A.P. Shotov. Electron-phonon interaction in $p$-type $\mathrm{Pb}_{1-\mathrm{X}} \mathrm{Sn}_{\mathrm{X}} \mathrm{Se}(\mathrm{x}=0,06)$ crystals // Phys. Tech. Semicond. (Russia), 9(9), p. 1824-1826 (1975).

10. Yu.I. Ravich, I.A. Smirnov, V.V. Tikhonov. About Lorentz number on n-PbTe solid solution PbTe-PbSe // Phys. Tech. Semicond. (Russia), 1(2), pp. 206-210 (1967).

11. E. Patley. Sulphide, selenide and telluride of lead // Materials, was use in semiconductor devices // Mir, M., pp. 271-305 (1968).

12. B.M. Askerov. Kinetic effects on semiconductors. Nauka, L. 112 p. (1970)

13. V.P.Tamarchenko, Yu.I. Ravich, L.Ya. Morgovski, I.N. Dubrovskaya. About Lorentz number and other kinetic coefficient on generate samples $\mathrm{PbTe}, \mathrm{PbSe}$ и $\mathrm{PbS} / /$ Phys. Solid State. (Russia), 11(11), pp. 3206-3213 (1969).

14. B.Ya. Moyzhes, Yu.I. Ravich. About scattering mechanism and role of interelectron scattering on $\mathrm{n}-\mathrm{PbTe}$ and any semimetals // Phys. Tech. Semicond. (Russia), 1(2), pp. 8195 (1967).

15. R.S. Allgaier, W.W. Scanlon. Mobility of Electrons and Holes in $\mathrm{PbS}, \mathrm{PbSe}$, and $\mathrm{PbTe}$ between Room Temperature and 4.2K // Phys. Rev, 111(4), pp. 1029-1037 (1958).

16. U. Schlighting, K.N. Gobrecht. The mobility of free carriers in PbSe crystals // J. Phys. Chem. Solids, 34, pp. 753-758 (1973).

17. D.M. Freik, M.O. Galuschak, L.I. Nykyruy, V.M. Klanichka, V.M. Shperun. Band structure, scattering mechanism and kinetic phenomena in $n$-PbTe crystals // Ukr. Phys. Journ., 46(4), pp. 499-502 (2001).

18. D.M. Freik, L.I. Nykyruy, L.Y. Mezhylovska, V.M. Klanichka, M.O. Galuschak, V.M. Shperun. Laws of dispersion and scattering mechanism in $n$-PbSe crystals // Ukr. Phys. Journ., 46(10), pp. 1344-1347 (2001).

19. D.M. Freik, L.I. Nykyruy, I.V. Kalytchuk, V.V. Nyzhnykevych. Feature of band structure and scattering mechanism in electronic crystals of lead chalcogenides // Naukovyy visnyk CNU: Physics. Electronics, 102, pp. 78-81 (2001).

20. D.M. Freik, L.I. Nykyruy, V.M. Klanichka, V.M. Shperun, R.I. Sobkovych, O.Ya. Dovgyy. Band structure and scattering mechanism on n-PbSe crystals at the $77 \mathrm{~K} / /$ Physics and Chemistry of Solid State, 1(2), pp.245-249 (2000).

21. L.I. Nykyruy. Laws of dispersion and transport phenomena on lead selenide electronic crystals // Physics and Chemistry of Solid State, 2(2), pp. 277-282 (2001).

22. D.M. Freik, L.I. Nykyruy, M.A. Ruvinskii, V.M. Shperun, V.V. Nyzhnykevych. Scattering mechanisms on n-types lead chalcogenides crystals // Physics and Chemistry of Solid State, 2(4), pp. 681-685 (2001).

23. D.M. Freik, L.I. Nykyruy, V.M. Klanichka, V.M. Shperun, I. Petkovich // Influence scattering mechanisms and band structure on thermal electric properties lead chalcogenides monocrystallines // IX International Conference on Thermal Electrics. Chernivtsy (Ukraine) (2000).

24. L.I. Nykyruy. Band structure and scattering mechanisms on lead chalcogenides // Materials of VIII International Conference on Physics and Technology of Thin Films (ICPPTFVIII), Ivano-Frankivsk (Ukraine), pp. 78-79 (2001).

25. D.M. Freik, L.I. Nykyruy, V.V. Nyzhnykevych. Scattering of electrons on lattice oscillation on lead chalcogenides crystals // Physics and Chemistry of Solid State, 3(2), pp. 215-220 (2002). 